\title{
Epidermal growth factor receptor expression in pre-invasive and early invasive bronchial lesions
}

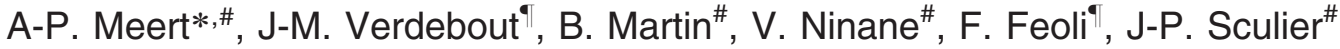

Epidermal growth factor receptor expression in pre-invasive and early invasive bronchial lesions. A-P. Meert, J-M. Verdebout, B. Martin, V. Ninane, F. Feoli, J-P. Sculier. (C) ERS Journals Ltd 2003.

ABSTRACT: The 1999 World Health Organization/International Association for the Study of Lung Cancer histological classification of preneoplastic bronchial lesions has been shown to be reproducible but little is known about its biological significance. The current study evaluated the correspondence between the morphological changes of the bronchial epithelium and epidermal growth factor receptor (EGF-R) expression.

Thirteen normal bronchial epithelia, 19 hyperplasia, 16 metaplasia, 10 mild dysplasia, one moderate dysplasia, 10 severe dysplasia (SD), 14 carcinoma in situ (CIS) and 11 microinvasive tumours were assessed. A global EGF-R score obtained by the sum of the positivity score plus the EGF-R staining intensity score was calculated for each lesion.

A global EGF-R score of $>5$ was reached only in one metaplasia, in six SD, in six CIS and in six microinvasive tumours. There was no difference in EGF-R expression between normal, hyperplastic and metaplastic epithelia versus mild dysplasia or between severe dysplasia versus CIS and microinvasive tumours but there was a statistically significant difference between mild versus severe dysplasia.

This study demonstrates that epidermal growth factor receptor expression rate changes with the stage of the bronchial lesion, increasing from normal epithelium to carcinoma in situ and microinvasive tumours with a statistically significant difference between mild versus severe dysplasia.

Eur Respir J 2003; 21: 611-615.

\author{
Correspondence: J-P. Sculier \\ Institut Jules Bordet \\ 1 , rue Héger Bordet \\ 1000-Bruxelles \\ Belgium \\ Fax: 3225343756 \\ E-mail: sculier@bordet.be
}

*Fond National de la Recherche Scientifique, ${ }^{\#}$ Dept of Internal Medicine and Laboratoire d'Investigation Clinique ęt d'Oncologie Expérimentale HJ Tagnon and Service of Pathology, Jules Bordet Institute, Bruxelles, Belgium.

Keywords: Epidermal growth factor receptor pre-invasive lesions

lung

Received: July 182002

Accepted after revision: December 122002

This research was supported by grants from the Fonds National de la Recherche Scientifique and from Les Amis de 1'Institut Bordet.
Lung cancer is the most common cause of death by malignancy in developed countries and less than $15 \%$ of the patients can be cured or have long-term survival. As tumour stage is the most significant prognostic factor for survival, there is considerable interest in early detection of lung cancer. It is also important to detect bronchial mucosal abnormalities that could be precursors for lung cancer. The precursors of squamous cell lung carcinoma are well known and include dysplasia and carcinoma in situ (CIS). Fluorescence bronchoscopy significantly increases the detection rate of these preneoplastic lesions [1-3] and a consensus (World Health Organization (WHO)/International Association for the Study of Lung Cancer (IASLC)) classification system of pre-invasive squamous lesions of the bronchial mucosa has recently become available [4]. This WHO/IASLC classification of pre-invasive squamous lesions of the bronchus developed in 1999 has been shown to be reproducible among pathologists with acceptable levels of inter- and intraobserver variations [5]. Although some studies suggest a parallel evolution of the biological and morphological abnormalities of these lesions [6], a better understanding of their biology is still necessary in order to have an optimal approach to their management and treatment.

Inhaled carcinogens (benzopyrenes, nitrosamines, etc.) may cause acquired genetic abnormalities to the mucosa of smokers and exsmokers. The malignant transformation of pulmonary epithelial cells is the result of a multi-step accumulation of genetic and molecular alterations involving proto-oncogenes, tumour suppressor genes, proliferation factors and apoptosis [7]. The genomic alterations can persist in bronchial lesions for months or years and the persistence and the regression of the molecular abnormalities have been correlated with the evolution of the lesions [6].

Among the multiple biomarkers that have been implicated in bronchial carcinogenesis, one of the erbB family receptors may be particularly interesting: the epidermal growth factor receptor (EGF-R). EGF-R plays a role in cell motility, adhesion, invasion and angiogenesis [8]. Some studies have indicated that the EGF-R signalling pathway may be involved in lung carcinogenesis $[9,10]$. In normal lung tissue, EGF-R is only expressed in the basal cell layer of the bronchial epithelium [10]. In nonsmall cell lung cancer (NSCLC), EGF-R overexpression is reported in $13-80 \%$ of tumours: $24-89 \%$ [11, 12] of squamous cell lung cancer and 23-46\% [13, 14] of adenocarcinoma. EGF-R gene amplification occurs in $\sim 6 \%$ of all NSCLC [15] and $28 \%$ of squamous cell lung cancers. In addition, a mutation, called EGF-RvIII, containing a constitutively active tyrosine kinase, has been documented. Finally, EGF-R expression seems to be associated with worse survival in NSCLC [16].

The aim of the present study was to evaluate the association between morphological changes of the bronchial epithelium and EGF-R expression in order to better understand the WHO/IASLC classification from a biological point of view.

\section{Materials and method}

\section{Study population}

To be eligible, patients had to have a minimum smoking exposure of 30 cigarette pack-yrs and/or a history of lung or 
head and neck cancer. Fluorescence bronchoscopy was performed under local anaesthesia and any area that appeared as abnormal in fluorescence was biopsied [17]. One-hundred and twenty-four eligible consecutive biopsies were reviewed from 124 different patients who had undergone laser-induced fluorescence (LIFE) bronchoscopy between February 1996 and October 2001. Ninety-eight patients were males and 26 females with a median age of 63 yrs (range 29-85). Thirty-six were current smokers, 67 were former smokers, and three were nonsmokers and smoking habits were unknown for 18 . Fifty-two had LIFE bronchoscopy for a follow-up of lung cancer (44 for NSCLC and eight for small-cell lung cancer), 26 for lung cancer detection, 22 for the pre-operative work-up of a lung [15] or head and neck cancer [7], 17 for the follow-up of a carcinoma in situ of the lung and seven for other various reasons. Several biopsies were performed for each patient; 154 normal epithelia, 32 hyperplasia, 71 metaplasia, 15 mild dysplasia, three moderate dysplasia, one severe dysplasia, eight CIS and 41 inflammatory tissues were observed. For statistical reasons, only the most severe lesion found in a given patient was evaluated and the patient was only included once in the study.

\section{Sample preparation and selection}

All the small biopsied tissues were routinely fixed in $10 \%$ neutral buffered formalin immediately following bronchoscopic procedure (for a minimum of $3 \mathrm{~h}$ and a maximum of $10 \mathrm{~h})$ and were embedded in paraffin. From each specimen block, 5- $\mu \mathrm{m}$ sections were cut from paraffin-embedded tissues and were deposited on SuperFrost Plus Slides (Menzel-Gläser, Braunschwein, Germany). All the haematoxylin eosin stained lesions were classified by one pathologist according to the 1999 histological WHO/IASLC classification of pre-invasive squamous lesions of the bronchus [4].

\section{Immunohistochemistry for epidermal growth factor receptor}

All the reagents had an analytical quality and were used without any preliminary purification. Methanol, citric acid, sodium citrate, tris(hydroxymethyl)aminomethane (TRIS) and hydrochloric acid were purchased from Merck (Darmstad, Germany).

Immunohistochemistry was performed according to a standard avidin-biotin-peroxidase complex [18] using a mouse monoclonal antibody directed against the external domain of EGF-R (immunoglobulin (Ig)G2a (NCL-EGFR; clone EGFR.113); Novocastra Laboratories, Newcastle-upon-Tyne, UK) giving a membrane staining.

The slides were deparaffinised in xylene and rehydrated in ethanol. The endogenous peroxidases were quenched by incubation in $0.3 \%$ hydrogen peroxide in methanol for $30 \mathrm{~min}$ at room temperature and the slides were rinsed twice in TRIS$\mathrm{HCl} 0.005 \mathrm{M}, \mathrm{NaCl} 0.9 \%$, $\mathrm{pH} 7.6$ for $10 \mathrm{~min}$. They were then submitted to antigen retrieval in citrate buffer $0.01 \mathrm{M}, \mathrm{pH} 7$ consisting of $3 \times 5$ min of microwave treatments at $650 \mathrm{~W}$. The slides were recooled during $25 \mathrm{~min}$ at room temperature. The antibodies anti-EGF-R (dilution 1/20, final titration $5 \mu \mathrm{g} \cdot \mathrm{mL}^{-1}$ ) were deposited and incubated during $60 \mathrm{~min}$ at $37^{\circ} \mathrm{C}$. All of the next steps were realised automatically at $37^{\circ} \mathrm{C}$ in the NexES system (Ventana Medical Systems, Tucson, AZ, USA). The complex between EGF-R and its antibodies was fixed with glutaraldehyde $\mathrm{NaCl} 0.9 \%$. The secondary biotinylated antibody was incubated for $8 \mathrm{~min}$. The slides were stained with a diaminobenzidine (DAB) detection kit (Ventana Medical Systems) and counterstained with haematoxylin.
Negative controls for EGF-R were carried out by omitting the primary antibody and also by substituting normal mouse IgG2a with primary antibody. The positive controls were a known EGF-R positive lung adenocarcinoma and placental tissue.

\section{Interpretation of immunohistochemistry}

Three observers (one pathologist, one oncologist and one biologist), trained in immunohistochemical examination of bronchial epithelial samples, independently evaluated the results of the immunohistochemical staining.

The bronchial lesion staining was scored as follows. 0: no staining or staining of basal cell layer only; $1+$ positive: staining of cells in the basal and suprabasal regions (maximum of the lower third of the epithelium thickness); $2+$ positive: staining of cells up to two-thirds of the epithelium thickness; $3+$ positive: staining cells in three-thirds of the epithelium thickness. The staining intensity was scored as follows. 0: no staining; 1: staining as normal bronchial epithelium; 2: staining stronger than normal epithelium; 3: staining as strong as the positive lung carcinoma control. The results were compared and discordant interpretations were resolved by review of the specific slides by the three observers at a multihead microscope. A global EGF-R score was obtained by the sum of the positivity score and the intensity score.

\section{Statistical assessment}

Bronchial lesions were grouped into four mains categories according to histology. Group 1: normal epithelium, hyperplasia and metaplasia; group 2: mild dysplasia; group 3: moderate dysplasia; group 4: severe dysplasia; and group 5: CIS and microinvasive tumours. In terms of EGF-R positivity, two categories were compared: 0 and $1+$ positive versus $2+$ and $3+$ positive lesions. In terms of intensity, the lesions were dichotomised into two groups: 0 and 1 intensity versus 2 and 3 intensity. In terms of global EGF-R score, the lesions were dichotomised between low scores of $<5$ and high scores of $\geqslant 5$ in order to have at least a positivity staining of two-thirds of the epithelium thickness with a grade 3 intensity or at least a positivity staining of three-thirds of the epithelium thickness with a grade 2 intensity. Comparisons were performed using bilateral Chi-squared tests or Fisher exact tests when needed because of the small number of lesions in some categories.

\section{Results}

Of the 124 eligible patients, 94 patients ( 74 males and 20 females) provided sufficiently large biopsies to evaluate EGF$\mathrm{R}$ expression. The median patient age was 63 yrs (range 29-86). Twenty-eight patients were current smokers, 49 were former smokers, and two were nonsmokers and smoking habits were unknown for 15 . The bronchoscopic procedure was performed in 20 patients for lung cancer detection, in 14 in the preoperative work-up of a bronchial (10 patients) or a head and neck (four patients) cancer, in 40 for follow-up of a previously treated lung cancer, in 14 for follow-up of CIS and in six for various reasons. Thirteen normal bronchial epithelia, 19 hyperplasia, 16 metaplasia, 10 mild dysplasia, one moderate dysplasia, 10 severe dysplasia, 14 CIS and 11 microinvasive tumours were found to be evaluable.

The immunostaining positivity results are shown in table 1 . EGF-R immunostaining was present in the basal cell layer in normal bronchial epithelium. It was present in the basal cell 
Table 1. - Epidermal growth factor receptor positivity according to the World Health Organization/International Association for the Study of Lung Cancer classification

$\begin{array}{ccccc}\text { Samples } & 0 & 1+ & 2+ & 3+ \\ \mathrm{n} & & \text { positive } & \text { positive } & \text { positive }\end{array}$

\begin{tabular}{lccllc}
\hline Normal & 13 & 13 & 0 & 0 & 0 \\
Hyperplasia & 19 & 19 & 0 & 0 & 0 \\
Metaplasia & 16 & 9 & 1 & 4 & 2 \\
Mild dysplasia & 10 & 10 & 0 & 0 & 0 \\
Moderate dysplasia & 1 & 1 & 0 & 0 & 0 \\
Severe dysplasia & 10 & 3 & 1 & 0 & 6 \\
CIS & 14 & 2 & 0 & 0 & 12 \\
Microinvasive tumour & 11 & 2 & 0 & 0 & 9 \\
\hline
\end{tabular}

0 : no staining or staining of basal cell layer only; $1+$ positive: staining of cells in basal and suprabasal regions (maximum of the lower third of the epithelium thickness); 2+ positive: staining of cells up to two-thirds of the epithelium thickness; $3+$ positive: staining cells in three-thirds of the epithelium thickness. CIS: carcinoma in situ.

layer but not in the more superficial layers of hyperplastic epithelium (fig. 1a). Seven out of 16 metaplasia showed a positive EGF-R staining involving more than basal cell layer but only two $(12 \%)$ were stained in the upper third of the epithelium. All the mild and moderate dysplasia had an immunostaining pattern similar to the normal epithelium. Seven out of 10 severe dysplasia had a positive EGF-R staining involving more than basal cell layer and six $(60 \%)$ of them stained all the epithelium thickness (fig. 1c). The majority of the CIS (86\%) (fig. 1d) and of the microinvasive tumours (82\%) (fig.1e) stained all the epithelium thickness. These results were statistically significant (Chi-squared $\mathrm{p}<0.001)$. When data were analysed according to groups as defined in the Statistical assessment section, there was no detectable difference between histological groups 1 and 2 $(\mathrm{p}=0.58)$ or between groups 4 and $5(\mathrm{p}=1)$ but there was a statistically significant difference between groups 2 and 4 $(\mathrm{p}=0.01)$.
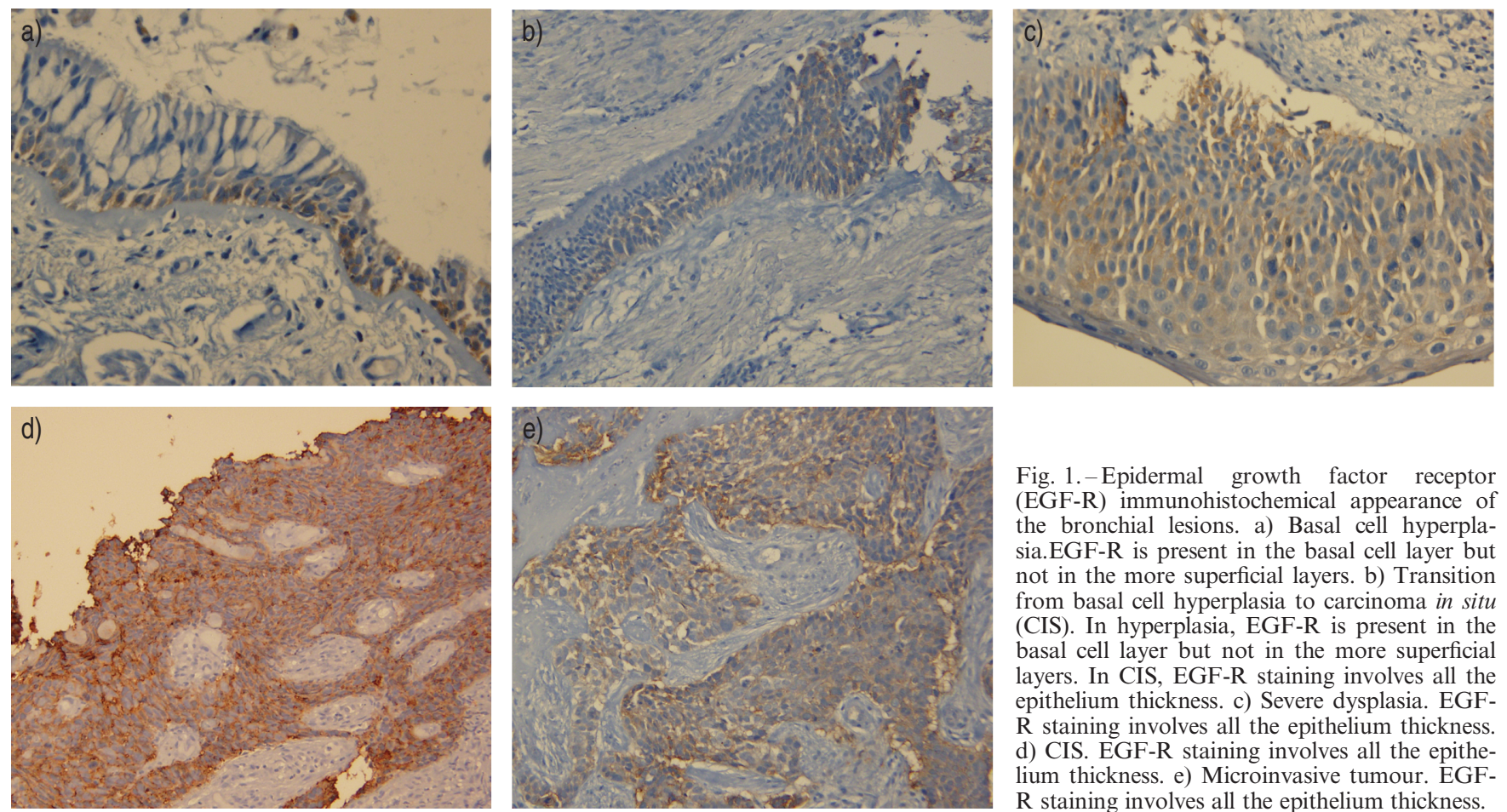

Table 2. - Epidermal growth factor receptor intensity according to the World Health Organization/International Association for the Study of Lung Cancer classification

\begin{tabular}{lccccr}
\hline & Samples n & 0 & 1 & 2 & 3 \\
\hline Normal & 13 & 0 & 13 & 0 & 0 \\
Hyperplasia & 19 & 4 & 15 & 0 & 0 \\
Metaplasia & 16 & 0 & 15 & 0 & 1 \\
Mild dysplasia & 10 & 0 & 10 & 0 & 0 \\
Moderate dysplasia & 1 & 0 & 1 & 0 & 0 \\
Severe dysplasia & 10 & 1 & 3 & 4 & 2 \\
CIS & 14 & 2 & 6 & 6 & 0 \\
Microinvasive tumour & 11 & 2 & 3 & 2 & 4 \\
\hline
\end{tabular}

0: no staining; 1: staining as normal bronchial epithelium; 2: staining stronger than normal epithelium; 3: staining as strong as the positive lung tumour control. CIS: carcinoma in situ.

A grade 2 or 3 intensity of the labelling was reached in one of the $16(6 \%)$ metaplasia, in none of the mild or moderate dysplasia, in six of the $10(60 \%)$ severe dysplasia, in six of the $14(43 \%)$ CIS and in six of the $11(55 \%)$ microinvasive tumours (Chi-squared test $\mathrm{p}<0.001)$ (table 2). There was no detectable difference between histological groups 1 and 2 $(\mathrm{p}=1)$ or between groups 4 and $5(\mathrm{p}=0.71)$ but there was a statistically significant difference between groups 2 and 4 $(\mathrm{p}=0.006)$.

A global EGF-R score of $\geqslant 5$ was reached in one $(6 \%)$ metaplasia, in all the mild and the only moderate dysplasia, in six $(60 \%)$ severe dysplasia, in six $(43 \%)$ CIS and in six $(55 \%)$ microinvasive tumours $(\mathrm{p}<0.001)$, with the same statistical results than intensity results between the histological groups (table 3).

\section{Discussion}

The current study demonstrates that EGF-R expression rate changes with the stage of the bronchial lesions, increasing from normal epithelium to CIS and microinvasive tumours 
Table 3. - Epidermal growth factor receptor (EGF-R) global score according to the World Health Organization/International Association for the Study of Lung Cancer classification

\begin{tabular}{lcccccccc}
\hline & Samples n & 0 & 1 & 2 & 3 & 4 & 5 & 6 \\
\hline Normal & 13 & 0 & 13 & 0 & 0 & 0 & 0 & 0 \\
Hyperplasia & 19 & 4 & 15 & 0 & 0 & 0 & 0 & 0 \\
Metaplasia & 16 & 0 & 9 & 1 & 4 & 1 & 0 & 1 \\
Mild dysplasia & 10 & 0 & 10 & 0 & 0 & 0 & 0 & 0 \\
Moderate dysplasia & 1 & 0 & 1 & 0 & 0 & 0 & 0 & 0 \\
Severe dysplasia & 10 & 1 & 2 & 1 & 0 & 0 & 4 & 2 \\
CIS & 14 & 2 & 0 & 0 & 0 & 6 & 6 & 0 \\
Microinvasive tumour & 11 & 2 & 0 & 0 & 0 & 3 & 2 & 4 \\
\hline
\end{tabular}

The global EGF-R score was obtained by the sum of the positivity score and of the intensity score. 0 : EGF-R positivity and intensity of $0 ; 1$ : EGF-R positivity or intensity of 0 and EGF-R intensity or positivity of $1 ; 2$ : EGF-R positivity and intensity of 1; 3: EGF-R positivity or intensity of 1 and EGF-R intensity or positivity of 2; 4: EGF-R positivity and intensity of 2 or EGF-R positivity or intensity of 1 and EGF-R intensity or positivity of 3; 5: EGF-R positivity or intensity of 2 and EGF$\mathrm{R}$ intensity or positivity of 3. 6: EGF-R positivity and intensity of 3; CIS: carcinoma in situ.

with a statistically significant cut-off between mild dysplasia and severe dysplasia. These results suggest that, at least when EGF-R expression is assessed, severe dysplasia behaves more like CIS than mild dysplasia.

YONEDA [9] also found that EGF-R positive cells extend upward throughout the epithelial layer, as the cytological atypia advance from metaplasia to dysplasia (not graded) and finally to CIS, in which the entire thickness of the epithelium is occupied by cytological atypical cells often staining for EGF-R. In this study, the author did not find any case of metaplasia with staining in the upper third. In contrast, RUSCH et al. [10] showed that EGF-R expression was not associated with specific preneoplastic lesions. In this study, EGF-R staining occurred as frequently in metaplasia as in dysplasia and CIS, although the staining was more intense and involved more of the superficial layers of the epithelium in dysplasia (not graded) and CIS. In 46 hyperplasia and 60 metaplasia specimens, PIYATHILAKE et al. [19] found a higher expression of EGF-R than in normal epithelium, with a higher expression in the basal cells than in the luminal cells, especially with a cytoplasmic staining. Conversely, the EGF$\mathrm{R}$ expression was higher in the cellular membrane in eight dysplastic lesions and in 60 lung cancers, suggesting a switch from cytoplasm to membrane from the dysplastic stage. Finally, in a trial evaluating the effect of treatment with 13-cis retinoic acid, KURIE et al. [20] demonstrated that EGF-R was higher in metaplastic biopsies than in normal epithelium $(p=0.02)$ and that in $50 \%$, reversal of bronchial metaplasia was associated with decreased EGF-R expression. It is difficult to compare the results of these authors with the results of the current study, first, because they did not use the last WHO/ IASLC classification and grouped together different grades of dysplasia, and secondly, because the cut-off to determine the EGF-R positivity was different. In premalignant lesions of the head and neck, GRANDIS et al. [21] found data similar to the present results for bronchial preneoplastic lesions: EGF-R levels were relatively low in mild dysplasia and increased with higher grades of dysplasia. Thus, in squamous respiratory tract lesions there seems to be an increased EGF-R staining in terms of EGF-R positive-stained cells percentage and/or in terms of localisation of the positive cells. A similar profile was demonstrated by HARPER et al. [22] in premalignant lesions of the prostate. This study showed a tendency to higher EGF-R and c-erbB-2 expression in high-grade prostatic intraepithelial neoplasia (PIN) and carcinoma than in low-grade PIN or benign hyperplastic prostate. Conversely, in urinary bladder, EGF-R expression was found to be equally frequent in normal and dysplastic urothelium [23].

The present results should be interpreted with consideration of the clinical data published by BOTA et al. [24]. They showed that in the absence of treatment, only $2 \%$ of nondysplastic biopsies and 3.5\% of low-grade dysplasia (mild and moderate) progress to high-grade lesions, while $37 \%$ of severe dysplasia and $87 \%$ of CIS remained high-grade lesions or relapsed at the same place after a transient regression during follow-up. On the basis of these findings, the authors recommend a simple follow-up for low-grade epithelial lesions without high-grade associated lesions but treatment for severe dysplasia persisting after 3 months and immediately for CIS. The clinical and biological factors influencing the progression or the regression of bronchial lesions remain unclear. For example, the persistence of tobacco exposure on the prevalence and severity of bronchial lesions is not clear.

Some limitations of the current study should be emphasised. First, the number of patients is limited although, to the present authors' knowledge, it is the largest series published on EGF-R in preneoplastic lung lesions. Moreover, the number of patients with moderate dysplasia was very low and only one of these was evaluable for EGF-R expression. This therefore had to be excluded from the statistical analysis. For several reasons, such as reproducibility, it was suggested in other studies that moderate dysplasia should be included in high-grade lesions. The current study could not examine the cut-off between moderate and severe dysplasia [5]. Secondly, immunohistochemistry was used to detect EGF-R expression but actually, there is no standardisation and validation of the immunohistochemical analyses for EGF-R detection. In a previous study, RUSCH et al. [25] showed that only uniform and intense levels of immunohistochemical staining in the lung tumour, compared to the uninvolved lung, correlated with overexpression at the ribonucleic acid (RNA) level; less intense staining or specific staining in few cells did not correlate as highly with overexpression at the total cellular RNA level. Therefore, the present authors choose a cut-off of 5 for the global EGF-R score in order to have a minimal intensity of 2 to consider the lesion as positive. A comparison with a molecular biology technique is therefore important in order to correctly interpret research results and to have a reproducible routine test. However, the advantages of immunohistochemistry are the maintenance of the tissue architecture and the possibility of localising the antigen on a small paraffin-embedded preneoplastic lesion. In addition, immunohistochemistry is probably the most applicable and cost-effective technique for routine use. This last point could become important if a clinical application with anti-EGF-R drugs takes place in the future. For example, all trans-retinoic acid treatment seems to repress EGF-R expression and to reduce EGF-dependent mitogenesis in NSCLC NNK (Nnitrosamine-4-(methylnitrosamino)-1-(3 pyridil)-1-butanone) transformed cells [26]. Finally, whereas EGF-R intensity and distribution are increasing from normal to microinvasive tumours, not all lesions of a given group behave equally in terms of EGF-R expression. Such intragroup heterogeneity may reflect the fact that EGF-R is not linked to a metaplastic phenomenon and a certain status of differentiation and proliferation but with other biological parameters that can drive oncogenesis and the rate of progression.

In conclusion, the current study, the first evaluating epidermal growth factor receptor expression in bronchial lesions classified according to the 1999 World Health Organization/International Association for the Study of Lung Cancer classification, suggests that, with reference to this marker, severe dysplasia behaves more as a carcinoma 
in situ than as a low-grade lesion. The frequently increased epidermal growth factor receptor expression in severe dysplasia, carcinoma in situ and microinvasive tumours as compared with normal, metaplastic epithelia, and mild and moderate dysplasia suggests that epidermal growth factor receptor could be a relatively early marker of malignant transformation. However, as the neoplastic progression requires additional factors, the study of other biological markers is necessary in order to determine whether a biological difference really exists between mild and severe dysplasia.

Acknowledgements. The authors would like to thank their biostatistician M. Paesmans and their technician M. Schoonackers for their assistance.

\section{References}

1. Hirsch FR, Prindiville SA, Miller YE, et al. Fluorescence versus white-light bronchoscopy for detection of preneoplastic lesions: a randomized study. J Natl Cancer Inst 2001; 93: 1385-1391.

2. Vermylen $\mathrm{P}$, Pierard $\mathrm{P}$, Roufosse $\mathrm{C}$, et al. Detection of bronchial preneoplastic lesions and early lung cancer with fluorescence bronchoscopy: a study about its ambulatory feasibility under local anaesthesis. Lung Cancer 1999; 25: $161-168$.

3. Lam S, Kennedy $\mathrm{T}$, Unger $\mathrm{M}$, et al. Localization of bronchial intraepithelial neoplastic lesions by fluorescence bronchoscopy. Chest 1998; 113: 696-702.

4. Travis WD, Colby TV, Shimosato Y, Corrin B, Brambilla E and in association with Sobin LH and pathologists from 14 other countries. WHO/IASLC classification of lung and pleural tumors. 3rd Edn. Berlin, Springer-Verlag, 1999.

5. Nicholson AG, Perry LJ, Cury PM, et al. Reproducibility of the WHO/IASLC grading system for pre-invasive squamous lesions of the bronchus: a study of inter-observer and intraobserver variation. Histopathology 2001; 38: 202-208.

6. Thiberville L, Payne P, Vielkinds $\mathrm{J}$, et al. Evidence of cumulative gene losses with progression of premalignant epithelial lesions to carcinoma of the bronchus. Cancer Res 1995; 55: 5133-5139.

7. Brambilla E, Brambilla C. Oncogenes and antioncogenes in the progression dynamics of lung cancer. Rev Mal Respir 2000; 17: 313-327.

8. de Jong JS, van Diest PJ, van der Valk P, Baak JP. Expression of growth factors, growth-inhibiting factors, and their receptors in invasive breast cancer. II: Correlations with proliferation and angiogenesis. J Pathol 1998; 184: 53-57.

9. Yoneda K. Distribution of proliferating-cell nuclear antigen and epidermal growth factor receptor in intraepithelial squamous cell lesions of human bronchus. Mod Pathol 1994; 7: 480-486.

10. Rusch V, Klimstra D, Linkov I, Dmitrovsky E. Aberrant expression of p53 or the epidermal growth factor receptor is frequent in early bronchial neoplasia and coexpression precedes squamous cell carcinoma development. Cancer Res 1995; 55: 1365-1372.

11. Sekine I, Takami S, Guang SG, et al. Role of epidermal growth factor receptor overexpression, K-ras point mutation and c-myc amplification in the carcinogenesis of non-small cell lung cancer. Oncol Rep 1998; 5: 351-354.

12. Gorgoulis V, Giatromanolaki A, Karameris A, et al. Epidermal growth factor receptor expression in squamous cell lung carcinomas: an immunohistochemical and gene analysis in formalin-fixed, paraffin-embedded material. Virchows Arch A Pathol Anat Histopathol 1993; 423: 295-302.

13. Sekine I, Takami S, Guang SG, et al. Role of epidermal growth factor receptor overexpression, K-ras point mutation and c-myc amplification in the carcinogenesis of non-small cell lung cancer. Oncol Rep 1998; 5: 351-354.

14. Tateishi M, Ishida T, Kohdono S, Hamatake M, Fukuyama Y, Sugimachi K. Prognostic influence of the co-expression of epidermal growth factor receptor and c-erbB-2 protein in human lung adenocarcinoma. Surg Oncol 1994; 3: 109113.

15. Reissmann PT, Koga H, Figlin RA, Holmes EC, Slamon DJ. Amplification and overexpression of the cyclin D1 and epidermal growth factor receptor genes in non-small-cell lung cancer. Lung Cancer Study Group. J Cancer Res Clin Oncol 1999; 125: 61-70.

16. Meert AP, Martin B, Delmotte P, et al. The role of EGF-R expression on patient survival in lung cancer: a systematic review with meta-analysis. Eur Respir $J$ 2002; 20: 975-981.

17. Vermylen P, Pierard P, Roufosse C, et al. Detection of bronchial preneoplastic lesions and early lung cancer with fluorescence bronchoscopy: a study about its ambulatory feasibility under local anaesthesis. Lung Cancer 1999; 25: $161-168$

18. King G, Payne S, Walker F, Murray GI. A highly sensitive detection method for immunohistochemistry using biotinylated tyramine. J Pathol 1997; 183: 237-241.

19. Piyathilake CJ, Frost AR, Manne U, et al. Differential expression of growth factors in squamous cell carcinoma and precancerous lesions of the lung. Clin Cancer Res 2002; 8: 734-744

20. Kurie JM, Shin HJ, Lee JS, et al. Increased epidermal growth factor receptor expression in metaplastic bronchial epithelium. Clin Cancer Res 1996; 2: 1787-1793.

21. Grandis JR, Tweardy DJ, Melhem MF. Asynchronous modulation of transforming growth factor alpha and epidermal growth factor receptor protein expression in progression of premalignant lesions to head and neck squamous cell carcinoma. Clin Cancer Res 1998; 4: 13-20.

22. Harper ME, Glynne-Jones E, Goddard L, Mathews P, Nicholson RI. Expression of androgen receptor and growth factors in premalignant lesions of the prostate. J Pathol 1998; 186: 169-177.

23. Wagner U, Sauter G, Moch H, et al. Patterns of p53, erbB-2, and EGF-r expression in premalignant lesions of the urinary bladder. Hum Pathol 1995; 26: 970-978.

24. Bota S, Auliac JB, Paris C, et al. Follow-up of bronchial precancerous lesions and carcinoma in situ using fluorescence endoscopy. Am J Respir Crit Care Med 2001; 164: 16881693.

25. Rusch V, Baselga J, Cordon-Cardo C, et al. Differential expression of the epidermal growth factor receptor and its ligands in primary non-small cell lung cancers and adjacent benign lung. Cancer Res 1993; 53: Suppl. 10, 2379-2385.

26. Lonardo F, Dragnev KH, Freemantle SJ, et al. Evidence for the epidermal growth factor receptor as a target for lung cancer prevention. Clin Cancer Res 2002; 8: 54-60. 